\title{
U-duality of Born-Infeld Theory
}

\author{
Gysbert Zwart \\ Spinoza Institute, Leuvenlaan 4, 3584 CE Utrecht \\ and \\ Institute for Theoretical Physics, University of Utrecht \\ Princetonplein 5, 3584 CC Utrecht, The Netherlands
}

AbSTRACT: String theory compactified on a three-torus possesses an $S L(5, \mathbf{Z})$ U-duality group. We investigate the realisation of this symmetry on the Born-Infeld theory on a three-brane, and discuss a U-duality covariant formulation of the BPS sector of the theory where the rank of the gauge group is treated on an equal footing with the fluxes.

\section{Introduction}

The simplest compactifications of string theories are those on tori. These preserve maximal supersymmetry, and furthermore they enjoy a large discrete symmetry, called U-duality. This U-duality group can be viewed as being generated by two distinct sets of symmetries (see e.g. [1. [1] and references therein).

The first is T-duality, a perturbative symmetry of string theory, i.e. it holds order by order in the string loop expansion. T-duality states that strings on circles of radius $R$ are in fact equivalent to strings on circles with inverse radii. In the identification, the roles of string momentum and winding states are interchanged. The T-duality group of string theory on a $d$-dimensional torus is $S O(d, d, \mathbf{Z})$.

The second contribution to the U-duality group follows from the observation that type IIA strings on a $d$-torus may alternatively be regarded as Mtheory on a torus of one dimension higher. This point of view makes obvious a geometric symmetry group $S L(d+1, \mathbf{Z})$ of the torus. From the string theory perspective, this is a non-perturbative symmetry: for instance, it interchanges non-perturba D0-branes with stringy momentum modes.

When these two groups are combined, they generate the U-duality groups, whose continuous versions have been known to exist for a long time as the hidden symmetries of supergravity theo- ries. For various torus dimensions $d$, they are listed in table

\begin{tabular}{|l|c|}
\hline$d$ & U-duality group \\
\hline 1 & $S L(2, \mathbf{Z})$ \\
\hline 2 & $S L(2, \mathbf{Z}) \times S L(3, \mathbf{Z})$ \\
\hline 3 & $S L(5, \mathbf{Z})$ \\
\hline 4 & $S O(5,5, \mathbf{Z})$ \\
\hline 5 & $E_{6(6)}(\mathbf{Z})$ \\
\hline 6 & $E_{7(7)}(\mathbf{Z})$ \\
\hline 7 & $E_{8(8)}(\mathbf{Z})$ \\
\hline
\end{tabular}

Table 1: U-duality groups for string theory compactified on various tori

centrate on the case $d=3$, where the U-duality group is $S L(5, \mathbf{Z})$.

The states in the theory transform in multiplets of the U-duality group. In particular this is the case for the $1 / 2$ BPS states. In type IIB strings on a three-torus these states are D3-branes, three different D1-branes (one for each cycle of the torus), three fundamental string winding modes and three momenta around the torus. These ten objects transform as an antisymmetric tensor under $S L(5)$. The $1 / 4$ BPS states, in which we tivifl be mainly interested, are realised as combinations of such $1 / 2$ BPS objects.

The symmetry under U-duality transformations also implies that the degeneracies of states related by such a transformation should coincide. In the case of $1 / 4$ BPS states this degeneracy 
can be calculated in perturbative string theory by considering a state with only momentum and winding quantum numbers. The degeneracy of a state with momentum vector $p_{i}$ and winding numbers $w_{i}$ is given by $D(p \cdot w)$, with $D(n)$ defined by the chiral string partition function:

$$
\sum D(n) q^{n}=256 \prod\left(\frac{1+q^{n}}{1-q^{n}}\right)^{8} .
$$

Since any other $1 / 4$ BPS state can be mapped to such a state, all 1/4 BPS degeneracies should be given by a similar expression.

The purpose of this work (reported in is to investigate the implications of the U-duality symmetry of the string theory on the Born-Infeld gauge theory living on a three-brane wrapping a torus. As we will see, the BPS states of the strings have an interpretation in terms of fluxes in the gauge theory on the three-brane. We will study the BPS sector of this Born-Infeld theory and, via BPS quantisation (following [4] termine the associated degeneracies of the BPS states in this gauge theory. These will turn out to be in accord with the string results.

\section{The Born-Infeld gauge theory on the three-brane}

We will focus the discussion on the seven-dimensional case, corresponding to string theory compactified on a three-torus, with U-duality group $S L(5, \mathbf{Z})$. On the type IIB string theory side, BPS states are built from ten distinct objects: three-branes wrapping the three-torus, fundamental and D-strings winding around three different one-cycles, and momentum modes in the three internal directions. The associated quantum numbers transform in the ten-dimensional representation of $S L(5)$.

In the gauge theory on a three-brane wrapping the torus, all these ten quantum numbers have an interpretation as fluxes. The relations are given in table $\overline{2}$. The number of D3-branes is of course related to the rank $N$ of the $U(N)$ gauge theory. The magnetic fluxes, the zero modes of the magnetic field $B_{i}=\frac{1}{2} \epsilon_{i j k} F_{i j}$, correspond to D-strings, whereas the S-dual electric fluxes take the role of fundamental strings. Finally the

\begin{tabular}{|c|c|}
\hline string theory & gauge theory \\
\hline D3-brane & rank $N$ \\
D1-branes & magnetic fluxes $B_{i}$ \\
fundamental strings & electric fluxes $E_{i}$ \\
momenta & gauge momenta $P_{i}$ \\
\hline
\end{tabular}

Table 2: Translation of string theory quantum numbers into gauge fluxes

momenta (i.e. the components of the integrated Poynting vector $E \wedge B$ ) simply translate to the string theory momenta around the torus.

To bring out the U-duality properties, it is convenient to organise these gauge theory quantities in an antisymmetric five by five tensor $M_{i j}$, as follows:

$$
M=\left(\begin{array}{ccccc}
0 & P_{3} & -P_{2} & E_{1} & B_{1} \\
-P_{3} & 0 & P_{1} & E_{2} & B_{2} \\
P_{2} & -P_{1} & 0 & E_{3} & B_{3} \\
-E_{1} & -E_{2} & -E_{3} & 0 & N \\
-B_{1} & -B_{2} & -B_{3} & -N & 0
\end{array}\right) .
$$

The $S L(5)$ acts on this matrix by conjugation. One can easily recognise the two subgroup $S L(3)$ and $S L(2)$. The former is the geometric symmetry on the torus and acts as such on the three vectors. It sits in the top lefthand block of the $S L(5)$ matrices. The two by two lower righthand block realises the $S L(2)$ which is the electromagnetic duality. It mixes electric and magnetic components. Both subgroups leave the rank $N$ untouched. We will discuss those transformations affecting the rank in the following.

The particular gauge theory on the D3-brane that we want to consider is the Born-Infeld gauge theory. The abelian version of its action is given by

$$
S_{B I}=\frac{1}{g_{s}} \int \sqrt{\operatorname{det}\left(G_{\mu \nu}+F_{\mu \nu}\right)} .
$$

The inverse string coupling in front of the action is typical of D-branes. We omit possible nontrivial $B$-field background contributions. The generalisation to higher rank is thought to be given by a symmetrised trace over the gauge group; this issue is not fully resolved yet however, for a discussion see e.g. [5] We will compute the Hamiltonian and BPS masses for the abelian case and assume the generalised result for arbitrary $N$. 
In order to calculate the Hamiltonian we need to introduce the electric field

$$
E^{i}=\frac{\delta \mathcal{L}}{\delta A_{i}} .
$$

It now turns out that the square of the Hamiltonian density $H$ can be expressed in a simple way in terms of the matrix $M_{i j}$ defined in equation $(2.1)$, as

$$
\begin{aligned}
H^{2} & =\frac{1}{g_{s}^{2}}\left(N^{2}+B^{2}\right)+E^{i} G_{i j} E^{j}+P_{i}\left(G^{-1}\right)^{i j} P_{j} \\
& =-\frac{1}{2} \operatorname{Tr} M^{2} .
\end{aligned}
$$

In the first line, we see the contribution from the D3 and D1-branes, with the characteristic coupling constant dependence, and then the terms corresponding to winding and momentum. The second line demonstrates that the Hamiltonian takes a very simple form in terms of the $S L(5)$ tensor $M$. (We can absorb the coupling, as well as any non-trivial background fields, in a fivedimensional metric).

This form suggests an $S L(5)$ covariant description of the theory. However, the matrix $M$ does not have arbitrary components, there exist relations between them. Remarkably we can write these relations again in an $S L(5)$ covariant form, as the constraint

$$
K^{i}=\frac{1}{8} \epsilon^{i j k l m} M_{j k} M_{l m}=0 .
$$

In components the five-vector $K^{i}$ is given by

$$
K=\left(N P_{i}-(E \wedge B)_{i},-P \cdot B, P \cdot E\right) .
$$

The first three components are precisely the definition of the Poynting vector, while the last two components are automatically zero whenever the first three are.

We are therefore led to consider an arbitrary matrix $M$, provided it satisfies the five-vector constraint $K=1 / 2(M \wedge M)=0$. At this point a major problem is of course that, to make the connection to gauge theory, while $E, B$ and $P$ may be position dependent, the rank $N$ should of course be a constant. We will turn to this in a moment.

Finally, we are interested in the BPS states of the theory. Again the BPS masses can be written in a nice form using the matrix $M_{i j}$. The BPS mass is a function of the ten charges, which are given by the zero modes of $M$. We write these as $m_{i j}=\int M_{i j}$. In terms of this matrix of fluxes, the BPS mass formula takes the form

$$
M_{B P S}^{2}=-\frac{1}{2} \operatorname{Tr} m^{2}+2|k| .
$$

Here $k$ is the zero mode equivalent of $K$, i.e. $k=$ $1 / 2(m \wedge m)$. Note that while the space dependent $K$ is automatically zero, $k$ is not. In fact $k=0$ only for $1 / 2$ BPS states, while $1 / 4$ BPS states have non-zero vector $k$. The BPS equations can be expressed in a covariant fashion in terms of $M$ and its zero-modes $m$ as well.

We will go on to quantise the space of BPS states, in order to try to determine the quantum degeneracies of the BPS states. We will first review the method of BPS quantisation introduced in [4] for the $U(N)$ Yang-Mills theory; then we will apply this to the Born-Infeld case.

\section{BPS quantisation of Yang-Mills theory}

In [4ind Hacquebord and Verlinde discussed the question of $S L(5)$ invariance of the BPS spectrum in the context of Yang-Mills theory on a torus. In the Yang-Mills theory we have the fields $A_{\mu}$, the vector potential, and six (adjoint) scalar fields $X^{I}$. The BPS equations depend on the fluxes of the configuration. In the simple case where only the momentum in the one-direction, $p_{1}$, and the rank $n$ are non-zero, we may gaugefix $A_{0}$ and $A_{1}$ to zero and then obtain the BPS equations

$$
\begin{gathered}
\left(\partial_{0}-\partial_{1}\right) A_{2,3}=0, \quad\left(\partial_{0}-\partial_{1}\right) X^{I}=0, \\
{\left[A_{i}, A_{j}\right]=\left[A_{i}, X^{I}\right]=\left[X^{I}, X^{J}\right]=0 .}
\end{gathered}
$$

These equations were recognised in $4 \overline{4}$ as the leftmoving sector of a matrix string theory. Due to the vanishing of the commutators, one can take all $n$ by $n$ matrices to be diagonal. At first sight this seems to imply that we have simply $n$ distinct left-moving theories on a string. However, in the periodicity conditions in the coordinate $x_{1}$ one may include a permutation of the eigenvalues,

$$
A_{i}\left(x_{1}+2 \pi\right)=S A_{i}\left(x_{1}\right) S^{-1},
$$


and similarly for the $X^{I}$, so that effectively one

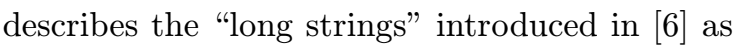
the twisted sectors of a conformal field theory on a symmetric product, with a total length $n$.

Hacquebord and Verlinde concentrated on the case where one has just one string of length $n$. In this case, quantisation of the theory restricted to the BPS configurations yields left-moving oscillators with a fractional moding, in multiples of $1 / n$. Then, to obtain a total momentum $p_{1}$ one should consider states with oscillator number $n p_{1}$, so that the degeneracy of such states is indeed given by $D\left(n p_{1}\right)$, the result from string theory.

For more general quantum numbers the degeneracies were argued to be the same in [4, total BPS degeneracies for the Yang-Mills $U(N)$ gauge theory therefore respect the U-duality group $S L(5, \mathbf{Z})$, at least in the single long string sector.

The essential point in this result is that in the subsector of the theory respecting the BPS conditions, the configurations reduce to strings. The length of these strings equals the rank of the gauge theory. We will now try to apply the same arguments to the Born-Infeld gauge theory. In the abelian case where the theory is well understood, we will again see the reduction to a string theory. For the non-abelian version, we will assume that similarly the equations reduce to those of a matrix string theory, so that effectively we can also here use the abelian BPS equations. We know that in the limit of large $N$, where the theory is adequately described by the Yang-Mills theory, this should be the case, but for general $N$ this remains an assumption essential for our result.

\section{BPS quantisation of the Born-Infeld theory}

For the supersymmetric Born-Infeld case we will now reexamine the situation. As explained above we will use the BPS equations found from the abelian Born-Infeld theory, and assume these to be valid for the non-abelian case, with the only alteration that the length of the domain on which the fields live is multiplied by the rank $n$.

Just as in the Yang-Mills case let us start from the easy case, where only the quantum num- bers associated to the rank, $n$, and the momentum in the one-direction, $p_{1}$, are non-zero. In this case the BPS equations are

$E_{2}=B_{3}, \quad E_{3}=-B_{2}, \quad E_{1}=B_{1}=P_{2}=P_{3}=0$.

If we insert the expressions for $E_{i}$ and $B_{i}$ in terms of the gauge field $A_{i}$ we again find the same equation as in the case of the Yang-Mills theory,

$$
\left(\partial_{0}-\partial_{1}\right) A_{i}=0
$$

and we are suppressing the six extra scalars $X^{I}$. From the fact that the equations are precisely the same we can of course conclude that here we have the same degeneracy as the one found in the previous situation. However, in order to be able to generalise to arbitrary fluxes, it is convenient to go through the calculation in a little more detail.

In order to quantise the theory in a lightcone gauge, we identify the electric field field with leftmoving string coordinates,

$$
E_{i} \sim \partial X_{i}
$$

enjoying the appropriate commutation relations

$$
\left[\partial X(\sigma), \partial X\left(\sigma^{\prime}\right)\right]=i \partial \delta\left(\sigma-\sigma^{\prime}\right) .
$$

From this relation to a string theory one can compute the degeneracy. However, let us step back and try to make the analogy to the string theory before the fixing to lightcone gauge. In order to do this we propose to identify the lightcone coordinates $\partial X^{ \pm}$with the rank, $N$, and the momentum, $P_{1}$. For the moment we therefore assume $N$ to be a real, fluctuating field, whose zero mode is the rank $n$. This implies imposing a commutation relation between the two quantities,

$$
\left[N, P_{1}\right]=i \partial \delta \text {. }
$$

In this notation, if we write down the constraint $K^{i}=0$ (equation $(2 . \overline{4})$ ), its only non-trivial component $K^{1}$ takes the form

$$
K^{1}=\partial X^{+} \partial X^{-}-\frac{1}{2} \partial X^{i} \partial X^{i}=0
$$

which we recognise as precisely the Virasoro constraint! Using the gauge symmetry generated by this constraint we may now fix $N=\partial X^{+}$to be 
a constant, $n$. This then determines $P^{1}=\partial X^{-}$ in terms of the other fields as

$$
n P_{1}=(E \wedge B)_{1} .
$$

In conclusion, by introducing an underlying pre-theory, in which the rank is allowed to be a fluctuating field, we manage to make contact to a string theory before the fixing of lightcone gauge. In this theory the constraint $K=0$ is interpreted as the Virasoro constraint. The original gauge theory is then identified with the lightcone gaugefixed version of this theory, where the rank is identified with the lightcone momentum.

So far we have only introduced some additional structure, which by fixing a gauge we again removed. The usefulness of this additional structure becomes clear when we consider the generalisation to arbitrary fluxes. To solve the problem for general fluxes, let us insert the expression in terms of the $\partial X$ 's from the previous discussion in the matrix $M_{i j}$ :

$$
M=\left(\begin{array}{ccccc}
0 & 0 & 0 & 0 & 0 \\
0 & 0 & \partial X^{-} & \partial X^{2} & -\partial X^{3} \\
0 & -\partial X^{-} & 0 & \partial X^{3} & \partial X^{2} \\
0 & -\partial X^{2} & -\partial X^{3} & 0 & \partial X^{+} \\
0 & \partial X^{3} & -\partial X^{2} & -\partial X^{+} & 0
\end{array}\right)
$$

Now, if $M_{i j}$ satisfies the Born-Infeld BPS equations, then, since these equations are covariant, an $S L(5)$ transformed $M_{i j}^{\prime}$ is a solution as well. If we therefore conjugate the matrix $M$ above with an appropriate $S L(5, \mathbf{Z})$ matrix, we obtain a new solution with arbitrary new zero-modes of the fields (fluxes). The entries of this new $M^{\prime}$ are all linear combinations of the $\partial X$, so the new electric and magnetic fields, as well as momenta and rank, are all functions of all the $\partial X$ 's. In particular, since the new rank $N^{\prime}$ is not anymore simply $\partial X^{+}$, it is no longer a constant. However, from the previous discussion we see that we may remedy this simply by making a gauge transformation generated by the constraint $K^{i}$, to fix the gauge so that again $N^{\prime}$ is a constant. Effectively, by performing a U-duality transformation we have gone out of the lightcone gauge, and one has to apply a compensating gauge transformation to reach a new configuration with constant rank. Since this is only a gauge transformation, it does not of course affect the degeneracy, so that this is indeed automatically U-invariant.

\section{Conclusions}

We have seen that Born-Infeld theory in $3+1$ dimensions can be naturally written in terms of U-covariant objects: the antisymmetric matrix $M_{i j}$, together with the five-vector of constraints $K^{i}=0$. The spectrum of BPS masses, as well as the BPS equations take a covariant form in terms of these quantities.

To study the degeneracies of the BPS states, we generalised the BPS quantisation applied to Yang-Mills theory in [4]. In the BPS sector, we saw that the theory reduced to a string theory, giving rise to the stringy degeneracies $D(n)$. Furthermore, we proposed a theory underlying the actual gauge theory in the BPS sector, in which the rank is treated on an equal footing as the other fields. In this formulation the constraint $K$ was identified with the generator of conformal transformations. Fixing this theory to lightcone gauge yields the actual gauge theory with constant rank $N$.

\section{References}

[1] N. Obers, B. Pioline, hep-th/9809039.

[2] C. Hofman, E. Verlinde, G. Zwart, JHEP 10 (1998) 020, hep-th/9808128.

[3] G. Zwart, JHEP 06 (1999) 010, hep-th/9905068.

[4] F. Hacquebord, H. Verlinde, Nucl.Phys. B508 (1997) 609, hep-th/9707179.

[5] A. Tseytlin, Nucl.Phys. B501 (1997) 41, hepth/9701125.

[6] R. Dijkgraaf, E. Verlinde, H. Verlinde, Nucl.Phys. B500 (1997) 43, hep-th/9703030. 